\section{Las denuncias de los afiliados de las empresas de medicina prepaga presentadas ante la Subsecretaría de Defensa del Consumidor en Argentina, 2000-2008}

\author{
Complaints by private health insurance policy- \\ holders to the Consumer Protection Bureau in \\ Argentina, 2000-2008
}

\author{
As denúncias de afiliados às empresas de planos \\ e seguros privados de saúde encaminhadas à \\ Subsecretaría de Defensa del Consumidor na \\ Argentina, 2000-2008
}

\begin{abstract}
This paper analyzes problems experienced by policy-holders of voluntary private health insurance plans in Argentina when insurance companies fail to comply with the Consumer Protection Code. The sample consisted of consumer complaints filed with the Consumer Protection Bureau and rulings by the Bureau from 2000 to 2008. One striking issue was recurrent non-compliance with services included in the Mandatory Medical Program and the companies' attempts to blame policy-holders. According to the study, the lack of an information system hinders scientific studies to adequately address the problem. Thus, a comparison with studies on health insurance in other Latin American countries highlighted the importance of such research, the relationship to health systems, constraints on use and denial of citizens' rights to healthcare, and the increasing judicialization of healthcare provision.
\end{abstract}

Health Maintenance Organizations; Legislation \& Jurisprudence; Consumer Advocacy
María José Luzuriaga 1

Hugo Spinelli 2

\section{Resumen}

El trabajo analiza los problemas de los afiliados a las empresas de medicina prepaga en Argentina cuando las mismas incumplen con la Ley de Defensa del Consumidor. El universo de estudio se conformó por las denuncias de los afiliados durante los años 2000-2008, ante la Subsecretaría de Defensa del Consumidor de la Nación, y por las disposiciones y laudos emitidos por la misma. Un aspecto conclusivo es la recurrencia de los incumplimientos en las prestaciones del Programa Médico Obligatorio y los argumentos expuestos por las empresas, centrados en la responsabilización de los afiliados. Se advierte que la falta de un sistema de información a nivel nacional impide la realización de estudios empíricos que reflejen adecuadamente el problema. La discusión se realiza a partir de estudios sobre los seguros privados de salud en otros países de la región. Sobre ello se observa la importancia de estudiar a las empresas de medicina prepaga, su articulación con el sistema de salud, y los procesos de limitación y negación de la ciudadanía, así como la creciente judicialización de la salud.

Sistemas Prepagos de Salud; Legislación \& Jurispridencia; Defensa del Consumidor 


\section{Introducción}

Los problemas en relación a la cobertura de los seguros privados de salud ha sido un tema recurrente en la investigación académica. La mayor parte de los trabajos fue realizado por investigadores norteamericanos 1,2,3. En América Latina el interés por la problemática ha sido creciente, principalmente, a partir de los procesos de reforma de los sistemas de salud en los años 90 4,5,6,7,8.

El presente trabajo busca ampliar el conocimiento sobre la problemática de las limitaciones y negaciones de cobertura, enfocándose en la situación argentina durante el periodo 2000-2008. Para tal fin, nos propusimos generar evidencia empírica que dé cuenta de los problemas denunciados por los usuarios de las empresas de medicina prepaga en nuestro país 9 .

La población cubierta por seguros privados, de acuerdo al censo de 2010 de Argentina, se estima en 6.222 .543 de personas ( $15 \%$ de la población), de las cuales el $67 \%$ serían afiliados indirectos, es decir, que acceden a la cobertura de una empresa de medicina prepaga a través de una obra social ${ }^{10}$. Debemos advertir que la información sobre las empresas de medicina prepaga es limitada y de baja calidad, ya que la misma es provista principalmente por las cámaras del sector y no existen registros oficiales 11. Diferentes estudios estiman la magnitud de las empresas de medicina prepaga en un rango de variación que va de 200 a 300 12,13,14. Una característica a destacar es la concentración en pocas empresas, siendo que cinco tienen entre el $50 \%$ y el $60 \%$ del total de los afiliados 12,13,14 -OSDE, Swiss Medical, Galeno, OMINT y Medicus 14. La primera de ellas tiene el $25 \%$ de la participación del sector con alrededor de 1,5 millones de afiliados en 200811.

Teniendo como referencia teórica la literatura disponible sobre el tema, el trabajo se estructuró en las siguientes preguntas: “¿Cuáles son los hechos denunciados por los usuarios de las empresas de medicina prepaga ante la Subsecretaría de Defensa del Consumidor de la Nación durante el periodo 2000-2008 en Argentina?” y, “icuáles son los argumentos de los representantes de la Subsecretaría de Defensa del Consumidor de la Nación, de las empresas de medicina prepaga y de los afiliados en el mismo periodo?"

La institucionalización de los derechos de los usuarios y consumidores en Argentina se alcanza entre 1993 -año en que se sanciona la Ley no 24.24015 de Defensa del Consumidor-y 1994 año en que se incorporaron esos derechos a través de la nueva Constitución Nacional (Artículo 42). La Secretaría de Comercio Interior 16 es la autoridad nacional de aplicación de la Ley no 24.240 y la Subsecretaría de Defensa del Consumidor -crea- da en el año 1998 como parte de la Secretaríainterviene y asesora a la misma en lo relativo a la ley mencionada.

Las empresas de medicina prepaga se encuentran reguladas por la Ley de Defensa del Consumidor, en tanto que comercializan un servicio que, en este caso, es el de la cobertura de salud a través del pago de una cuota mensual. Belmartino 17 (p. 24-5) define dichas empresas como "organizaciones de seguro privado que cubren a la población de mayor ingreso. En algunos casos surgen de mecanismos de integración hacia atrás de grandes hospitales privados $u$ organizaciones gremiales médicas". A su vez la reforma llevada a cabo durante los años 90 permitió el acceso de las empresas al sistema de obras sociales, a partir de la desregulación del aporte de los afiliados, permitiendo captar importantes subsidios públicos en la medida que el convenio con una obra social les posibilitó tener acceso a los recursos del Fondo Solidario de Distribución 18. A su vez, las mismas se convirtieron en un sector de gran interés para el capital financiero nacional e internacional 17,18,19,20. Dentro de sus estrategias de captación de clientes desarrollaron una "gama de planes con posibilidad de adaptarse a la capacidad adquisitiva o a la voluntad de cada cliente. Las diferencias en el monto suponen obviamente diferencias de acceso a servicios" 4 (p. 20).

Respecto a la normativa que regula a este sector en Argentina, en 1996, a partir de la Resolución no 247/96 del Ministerio de Salud y Acción Social, entra en vigencia el Programa Médico Obligatorio (PMO). Dicho programa fue en un primer momento el régimen de asistencia obligatoria sólo para las obras sociales comprendidas en las Ley no 23.660. En el año 1997 se extendió a las empresas de medicina prepaga a través de la Ley no 24.754. Allí se establece una serie de prestaciones que son de cumplimiento obligatorio para las empresas. También se define que no se podrán establecer períodos de carencia ni coseguros o copagos, fuera de lo expresamente indicado en dicho programa. En el año 2011 se aprobó la Ley no 26.28221 que regula la actividad de las empresas de medicina prepaga, con la cual se busca " $l i$ mitar los efectos de ciertas prácticas empresariales que habían dificultado a los afiliados el acceso a prestaciones de salud, como también el control desde el Estado de estas entidades" 22 (p. 3).

Las tensiones entre la legislación y la efectiva garantía de acceso a los servicios de salud ofrecidos por las empresas de medicina privada se evidencian también en estudios realizados en otros países de la región como Brasil 6,8,23,24,25, Chile 26,27,28 y Colombia 29,30. En el caso de Brasil, los estudios muestran las limitaciones de las empresas para ofrecer los servicios de salud contra- 
tados por los usuarios, así como las limitaciones regulatorias para garantizar el cumplimiento de los contratos 8,23,24,25. Se destacan los problemas vinculados a la negación o restricción de la cobertura de salud, aumentos abusivos de cuotas y rescisión unilateral de contratos 8,24,25. El Poder Judicial es el principal espacio de reivindicación de los afiliados a los planes y seguros de salud 24, evidenciando las debilidades de las instancias regulatorias ${ }^{8}$.

En Chile 26,27,28 y Colombia 29,30 también se observan conductas irregulares por parte de las empresas privadas de salud, así como problemas regulatorios a través de un creciente proceso de judicialización. En Colombia 29 el crecimiento de la judicialización, tanto en el sistema público, como privado, se debió principalmente a la negativa de cobertura por parte de las empresas. A partir de la gravedad de la situación, la Corte decidió realizar el dictado de la tutela T-760/2008 29. En la misma se enfatiza sobre la necesidad de abordar el problema desde un enfoque estructural, ya que los mecanismos regulatorios del Estado no han conseguido garantizar el derecho a la salud y las compañías de seguros siguen sin asimilar los principios reiteradamente establecidos por la Corte 29.

Adquieren particular relevancia los resultados de los estudios citados, debido a que en $\mathrm{Ar}$ gentina existen pocos estudios empíricos en el campo académico 31 que nos permitan dialogar con los resultados obtenidos en nuestro estudio.

\section{Metodología}

Para dar cuenta de los problemas existentes entre los usuarios y las empresas de medicina prepaga se utilizó la información provista por la Subsecretaría de Defensa del Consumidor de la Nación a través de cuatro de sus unidades organizativas: la Dirección de Defensa del Consumidor, la Dirección de Actuaciones por Infracción, el Sistema Nacional de Arbitraje de Consumo, y el Centro de Atención y Orientación a Consumidores (Tabla 1).

Tabla 1

Matriz de datos utilizada en el estudio.

\begin{tabular}{|c|c|c|c|}
\hline Unidades de análisis/periodo & Variables & Subuniversos & Fuentes de datos \\
\hline $\begin{array}{l}\text { Denuncias sobre empresas de } \\
\text { medicina prepaga, 2000-2008 }\end{array}$ & Motivo de la denuncia & 308 & $\begin{array}{l}\text { Base de datos de la Dirección } \\
\text { de Defensa del Consumidor }\end{array}$ \\
\hline $\begin{array}{l}\text { Disposiciones sobre empresas } \\
\text { de medicina prepaga cuyas } \\
\text { instancias de conciliación } \\
\text { fracasaron, } 2000-2008\end{array}$ & $\begin{array}{l}\text { Argumentos y fundamentos de } \\
\text { las EMP durante la audiencia y en } \\
\text { los descargos } \\
\text { Argumentos y fundamentos de } \\
\text { la Dirección de Actuaciones por } \\
\text { Infracción para sancionar a las } \\
\text { empresas de medicina prepaga }\end{array}$ & 45 & $\begin{array}{l}\text { Disposiciones de la Dirección } \\
\text { de Actuaciones por Infracción }\end{array}$ \\
\hline $\begin{array}{l}\text { Reclamos sobre empresas de } \\
\text { medicina prepaga, } \\
2000-2008\end{array}$ & Motivo del reclamo & 601 & $\begin{array}{c}\text { Base de datos del Sistema } \\
\text { Nacional de Arbitraje de } \\
\text { Consumo }\end{array}$ \\
\hline $\begin{array}{l}\text { Laudos sobre empresas de } \\
\text { medicina prepaga, 2000-2008 }\end{array}$ & $\begin{array}{c}\text { Argumentos y fundamentos } \\
\text { de las empresas de medicina } \\
\text { prepaga por negar la prestación } \\
\text { durante la audiencia y los } \\
\text { descargos } \\
\text { Argumentos y fundamentos } \\
\text { dados por los árbitros del } \\
\text { Sistema de Arbitraje de consumo } \\
\text { para exigir a la empresas de } \\
\text { medicina prepaga la solución del } \\
\text { problema reclamado }\end{array}$ & 60 & $\begin{array}{c}\text { Laudos del Sistema Nacional de } \\
\text { Arbitraje de Consumo }\end{array}$ \\
\hline $\begin{array}{l}\text { Consultas telefónicas sobre } \\
\text { empresas de medicina } \\
\text { prepaga, 2006-2008 }\end{array}$ & Motivo de las consultas & 10.042 & $\begin{array}{l}\text { Base de datos del Centro de } \\
\text { Atención y Orientación } \\
\text { a Consumidores }\end{array}$ \\
\hline
\end{tabular}

Fuente: elaboración propria. 
La información es de acceso público 32 y fue solicitada a la autoridad del organismo, ya que no se encuentra disponible en el sitio web de la Subsecretaría. El proceso de autorización y de obtención de los documentos analizados se vio facilitado por la vinculación laboral a la organización de uno de los autores del estudio.

Es preciso destacar que el universo analizado no corresponde a la totalidad de las denuncias realizadas por los usuarios de las empresas de medicina prepaga en el país. No fueron consideradas aquellas realizadas ante la Superintendencia de Servicios de Salud (con la sanción de la Ley no 26.282 de 2011, la misma puede recibir las denuncias de todos los afiliados a las empresas de medicina prepaga, siendo que en el periodo estudiado solo podía recibir las de los afiliados indirectos). Tampoco fueron analizadas las denuncias presentadas ante las oficinas provinciales y municipales, como ante las asociaciones de consumidores, ya que el país no cuenta con un sistema de información que concentre el total de las mismas. Por las limitaciones de información mencionadas elegimos la autoridad de mayor jerarquía administrativa a nivel nacional para la aplicación de la normativa bajo estudio. Hecho que debe ser tenido en cuenta en el momento de hacer la lectura de los resultados y que explica el bajo número de denuncias percibidas.

\section{Resultados}

En relación a los datos cuantitativos, los motivos más denunciados ante la Dirección de Defensa del Consumidor durante el periodo bajo estudio fueron la "falta o incompleta cobertura de prestación" con un $56 \%$ y el "aumento de cuota" con un $20 \%$ (Tabla 2). Dentro de la categoría "falta o incompleta cobertura de prestación”, la mayoría de las denuncias se refirieron a medicamentos $(26,9 \%)$, tratamientos $(24,7 \%)$ y operaciones $(20,4 \%)$ (Tabla 3). En el caso del Sistema Nacional de Arbitraje de Consumo los principales reclamos fueron el "incumplimiento de contrato" (35 \%) y la "falta o incompleta cobertura de prestación” con un 27\% (Tabla 4).

Entre los motivos más consultados a la línea gratuita de la Subsecretaría de Defensa del Consumidor, durante el periodo 2006-2008, encontramos consultas sobre "información general" (37\%), con un $18 \%$ consultas referidas al "aumento de cuotas"; y con un $12 \%$ a "la falta o incompleta cobertura de prácticas incluidas en el PMO” (Tabla 5). Si bien no formaron parte de nuestro subuniverso las consultas telefónicas realizadas en el periodo 2001-2006 -no obtuvimos los registros de las mismas-, identificamos a partir de la publicación de la revista institucional 33 que entre los años 2001 a 2006 las empresas de medicina prepaga se ubicaron entre el primer y cuarto rubro más consultado dentro del universo total de las consultas atendidas.

Otra dimensión analizada fue el posicionamiento de las empresas de medicina prepaga registrado tanto en los laudos como en las disposiciones-, respecto a los problemas denunciados por los afiliados. Entre los mismos se destacaron argumentos con énfasis en la falta de responsabilidad de los afiliados tales como: "la responsabilización del afiliado por estar mal informado" o "haber ocultado una enfermedad preexistente" (15 casos); "el no reconocimiento de prácticas establecidas en el PMO" (nueve casos); "la existencia de la denuncia en sede judicial" (cuatro menciones); "ya haber cumplido con la irregularidad denunciada” (tres menciones); y "la alusión a la figura mutual o asociación sin fines de lucro" (tres menciones) que las exime de la aplicación de la normativa por la que fueran denunciadas.

En relación a los argumentos de los representantes del Estado ante los hechos denunciados se utilizaron argumentos que enfatizaron "la asimetría de la relación contractual”; "la asimetría de la información"; "la responsabilidad de la empresa en función de la entidad del bien que tutela"; y "la limitada autonomía del afilado en el momento de contratar". Respecto a "la asimetría de la relación contractual”, identificamos en ocho disposiciones, un extracto que remarca el poder desigual de negociación, vinculado a la disparidad del conocimiento existente entre la empresa y el potencial afiliado y la alteración de lo que se considera la autonomía de la voluntad para contratar, definida en el Código Civil. Asimismo, para destacar la responsabilidad del bien tutelado por la empresa denunciada también se utilizó teoría jurídica en 17 disposiciones.

Otro eje en el cual se fundamentó la sanción a las empresas denunciadas fue en la responsabilidad de las mismas respecto a las características del bien tutelado. Para destacar este aspecto se utilizó jurisprudencia. Uno de los fallos que aparece citado en 17 disposiciones por la Dirección de Actuaciones por Infracción es el fallo Echeverri/Omint S.A. 34 que se originó a partir de la presentación de un recurso extraordinario por parte de un afiliado a una empresa de medicina prepaga, Omint S.A., que ante la pérdida del empleo solicitó el mantenimiento de la cobertura médica, haciéndose cargo personalmente del costo del servicio. El afiliado era portador de VIH/SIDA y por ese motivo la empresa se rehusó a aceptar la solicitud.

Los tratados internacionales también sustentaron las decisiones de la Dirección de Actuacio- 
nes por Infracción en 14 de los casos denunciados por los afiliados de empresas de medicina prepaga. Por último, encontramos que se utilizó doctrina jurídica para fundamentar la responsabilidad de la empresa de medicina prepaga en relación a su obligación de cumplir con aquellas prestaciones negadas a los afiliados referidas a enfermedades preexistentes. En la misma se destaca la obli- gatoriedad, por parte de la empresa de medicina prepaga, de hacer todos los estudios necesarios antes de celebrar el contrato (17 casos).

Los resultados presentados muestran una recurrencia y persistencia durante el periodo estudiado, en cuanto a los problemas denunciados por los usuarios, expresando una tenue o frágil regulación por parte del Estado en este sector.

Tabla 2

Frecuencia de los motivos de las denuncias sobre empresas de medicina prepaga presentadas por los usuarios ante la Dirección de Defensa del Consumidor. Argentina, 2000-2008.

\begin{tabular}{lcc}
\hline Motivos de incumplimiento de las denuncias & $\mathbf{n}$ & $\%$ \\
\hline Falta o incompleta cobertura de prestación & 174 & 56,5 \\
Aumento de cuota & 61 & 19,8 \\
Reintegros & 17 & 5,5 \\
Baja injustificada & 13 & 4,2 \\
Incumplimiento de Resolución 56 y 60 & 6 & 1,9 \\
Facturación incorrecta & 4 & 1,3 \\
Solicitud de cambio de plan & 4 & 1,3 \\
Incumplimiento de contrato & 3 & 1,0 \\
Pedido de afiliación & 3 & 1,0 \\
Incumplimiento de Resolución 9 y 26 & 3 & 1,0 \\
Otros & 10 & 3,2 \\
Sin datos & 10 & 3,2 \\
Total & 308 & 100,0
\end{tabular}

Fuente: elaboración propia, en base a la información provista por la Dirección de Defensa del Consumidor, perteneciente a la Subsecretaría de Defensa del Consumidor de la Nación.

Frecuencia de los motivos de las denuncias sobre las empresas de medicina prepaga, iniciadas por falta de cobertura o cobertura incompleta de una prestación de salud, presentadas por los usuarios ante la Dirección de Defensa del Consumidor. Argentina, 2000-2008.

\begin{tabular}{llc}
\hline Motivos de falta o incompleta cobertura de prestación & $\mathbf{n}$ & $\%$ \\
\hline Falta o incompleta cobertura de medicamentos & 25 & 26,9 \\
Falta o incompleta cobertura de tratamiento & 23 & 24,7 \\
Falta o incompleta cobertura de operación & 19 & 20,4 \\
Falta o incompleta cobertura de embarazo y/o parto & 8 & 8,6 \\
Falta o incompleta cobertura de estudios & 6 & 6,5 \\
Falta o incompleta cobertura de insumos para operación & 5 & 5,4 \\
Falta o incompleta cobertura de prótesis, ortesis & 3 & 3,2 \\
Falta o incompleta cobertura de traslado & 3 & 3,3 \\
Falta o incompleta cobertura de consultas post-operatorio & 1 & 1,1 \\
Total & 93 & 100,0 \\
\hline
\end{tabular}

Fuente: elaboración propia, en base a la información provista por la Dirección de Defensa del Consumidor, perteneciente a la Subsecretaría de Defensa del Consumidor de la Nación. 
Frecuencia de los motivos de reclamos sobre las empresas de medicina prepaga presentados ante el Sistema Nacional de Arbitraje de Consumo. Argentina, 2000-2008.

\begin{tabular}{|c|c|c|}
\hline Motivos del reclamo & $\mathbf{n}$ & $\%$ \\
\hline Incumplimiento de contrato & 211 & 35,1 \\
\hline Falta o incompleta cobertura de prestación & 162 & 27,0 \\
\hline Facturación incorrecta & 59 & 9,8 \\
\hline Aumento de cuota & 54 & 9,0 \\
\hline Falta de información & 36 & 6,0 \\
\hline Negación de reintegros & 28 & 4,7 \\
\hline Baja injustificada & 21 & 3,5 \\
\hline Otros & 27 & 4,5 \\
\hline Sin datos & 3 & 0,5 \\
\hline Total & 601 & 100,0 \\
\hline
\end{tabular}

Fuente: elaboración propia, en base a los datos provistos por el Sistema Nacional de Arbitraje de Consumo, perteneciente a la Subsecretaría de Defensa del Consumidor de la Nación.

Tabla 5

Frecuencia de los motivos consultados sobre las empresas de medicina prepaga a la línea gratuita de la Subsecretaría de Defensa del Consumidor. Argentina, 2006-2008.

\begin{tabular}{|c|c|c|}
\hline Motivos consultados & $\mathbf{n}$ & $\%$ \\
\hline Información general (consultas por datos de oficinas de defensa del consumidor, u otros organismos) & 3.767 & 37,5 \\
\hline Aumentos de cuotas por razones no contractuales & 1.849 & 18,4 \\
\hline Falta de cobertura o cobertura incompleta, prácticas incluidas en el PMO & 1.242 & 12,4 \\
\hline Carencia, preexistencia o copagos que no corresponden & 300 & 3,0 \\
\hline Incumplimiento de notificación (60 días) & 283 & 2,8 \\
\hline Cobertura de \% de medicamentos incorrecta & 252 & 2,5 \\
\hline Incumplimiento prestaciones pacientes crónicos & 199 & 2,0 \\
\hline Baja compulsiva/engañosa (edad-crónicas-seniles) & 191 & 1,9 \\
\hline Incumplimiento de plan materno infantil & 172 & 1,7 \\
\hline Sobrefacturación & 183 & 1,8 \\
\hline Incumplimiento de cobertura de prótesis u ortesis & 163 & 1,6 \\
\hline Incumplimiento cobertura oncológica & 146 & 1,5 \\
\hline Incumplimiento promesas contractuales/publicitarias/promociones & 122 & 1,2 \\
\hline Otros & 1.173 & 11,7 \\
\hline Total & 10.042 & 100,0 \\
\hline
\end{tabular}

PMO: Programa Médico Obligatorio.

Fuente: elaboración propia a partir de los datos obtenidos del sistema de información del Centro de Atención y Orientación a Consumidores de la Subsecretaría de Defensa del Consumidor de la Nación.

\section{Discusión}

A continuación vamos a discutir nuestros resultados con estudios empíricos que abordan el tema en nuestro país 31 y en otros países de la región 8,24,25,26,27,28,29,30.

En Argentina, en lo referente a la cobertura de servicios de salud limitada o negada por las em- presas de medicina prepaga, identificamos resultados similares a los nuestros en el estudio de Abramovich \& Pautassi 31. Los mismos encuentran el acceso a medicamentos y a tratamientos entre los principales motivos de los fallos de los Tribunales y la Corte Suprema de Justicia de Argentina, referidos a empresas de medicina prepaga y obras sociales 31 . A su vez, en los fallos es- 
tudiados por Abramovich \& Pautassi 31 se observa que los fundamentos, tanto de la Corte Suprema, como de los tribunales, colocan el derecho a la salud sobre el derecho de propiedad, citando en ambos casos jurisprudencia, como los pactos e instrumentos internacionales de derechos humanos. Un último aspecto a comparar se refiere a que en ambos estudios la rescisión unilateral del contrato también fue uno de los problemas denunciados por los afiliados a las empresas de medicina prepaga.

En Brasil 8,24,25, Chile 26,27,28 y Colombia 29,30 se realizaron estudios que identificaron resultados similares a los nuestros, respecto al tipo de problemas denunciados por los usuarios de las empresas de medicina prepaga, los argumentos dados por las mismas y por el Estado.

En relación al tipo de problemas, un estudio sobre las acciones con pedido de tutela anticipada en los Tribunales de Justicia de Río de Janeiro y de San Pablo 24, identificó las restricciones de cobertura, aumentos abusivos, y las recisiones unilaterales de contrato entre las principales causas que explican el aumento de reclamos ante la Justicia por parte de los afiliados a planes y seguros privados de salud ${ }^{24}$. Del mismo modo, en nuestro estudio las categorías "falta o incompleta cobertura de prestación”, "incumplimiento de contrato" y "el aumento de cuota” están entre los principales motivos denunciados, tanto ante la Dirección de Defensa del Consumidor, como ante el Sistema de Arbitraje de Consumo (tablas 2 y 4). La categoría "baja injustificada”, al igual que en el estudio citado -en el cual dicha categoría se define como rescisión unilateral del contrato-, aparece entre los motivos denunciados por los usuarios en nuestro estudio, si bien no se encuentra entre los principales.

En relación a los argumentos dados por las empresas y las respuestas del Estado, un estudio realizado por Scheffer 8 en San Pablo identificó resultados similares a los nuestros. Respecto a los argumentos de las empresas de medicina prepaga, observó que la defensa de las mismas se centró en la responsabilización al usuario. Así como nosotros identificamos entre los principales argumentos de las empresas "la responsabilización al afiliado por estar mal informado o por haber ocultado enfermedad preexistente" y "la prestación denunciada no establecida en el PMO"; el estudio de Scheffer 8 identificó entre los principales argumentos de negativa de cobertura de servicios "la existencia de cláusula excluyente en el contrato" y "el tratarse de una enfermedad preexistente" 8 . Por otra parte, resulta relevante advertir que en los fundamentos dados por las diferentes estructuras del Estado, sea en la esfera judicial en el caso de Brasil, como en el ejecutivo en nuestro estudio, se centraron en las condiciones contractuales altamente asimétricas que favorecen a la empresa y colocan al usuario en una situación desfavorable, en circunstancias de alta vulnerabilidad para este último.

También observamos argumentos semejantes, en cuanto a los fundamentos de las empresas de medicina prepaga, sobre las negativas de las prestaciones en un estudio 25 que analizó las denuncias de los beneficiarios de planes y seguros de salud ante la Agencia Nacional de Salud Suplementaria (ANS) en Río de Janeiro. El mismo destaca entre los motivos argumentados por las operadoras para justificar la negativa a dar cobertura asistencial, la ausencia de una red prestadora, y el ser un procedimiento que no se encontraba en el mínimo de prestaciones obligatorias a ser otorgadas (ROL) 25. En nuestro caso, el argumento referido a "el no reconocimiento de prácticas establecidas en el PMO" fue el segundo argumento más utilizado por las empresas de medicina prepaga, siendo que la "ausencia de una red prestadora” no apareció entre los principales.

Si bien nosotros no podemos realizar una asociación entre las principales enfermedades que explican los mayores índices de enfermedad y de mortalidad, respecto a las enfermedades por las cuales las empresas de medicina prepaga negaron su cobertura a los usuarios, el estudio de Scheffer 8 (p. 138) consigue realizar esa asociación y afirma que las principales negativas de cobertura de salud denunciadas "se relaciona con los problemas de salud y enfermedad responsables por los mayores índices de enfermedad y muerte de la población". Asimismo, el perfil de coberturas negadas se relacionó con aquellas situaciones clínicas en las cuales la demora en la asistencia o el diagnóstico resultaba determinante 8 .

Por otra parte, también nuestros resultados pueden ser comparados con estudios realizados en otros países de la región, como son los casos de Chile 26,27,28 y Colombia 29,30. En 2013 un informe de la Superintendencia de Salud de Chile 26 mostró el alto nivel de reclamos de los usuarios de los seguros privados de salud administrados por las Instituciones de Salud Previsional (ISAPRES). Entre los principales motivos de reclamo se encontraron "el alza de precio base", "la atención de urgencia" y "la negativa de cobertura por preexistencia" 26. Dichos problemas también son los principales temas judicializados en el país durante los últimos años 27,28. Nuevamente, encontramos similitudes con los resultados que obtuvimos, al observar entre los motivos que aparecen en nuestros subuniversos -denuncias, reclamos y consultas- "la falta o incompleta cobertura de la prestación” y "el aumento de cuota". 
En cuanto al sector privado de la salud en Colombia, se observa un proceso creciente de litigios entre los usuarios de planes y seguros de salud y las empresas privadas de salud 29,30. Así como en nuestro estudio las principales prestaciones negadas o limitadas fueron las referidas a medicamentos, tratamientos y operaciones (tabla 3), se observa que la mayoría de las tutelas judiciales en el estudio de Colombia fueron iniciadas por negativas a las prestaciones de tratamientos que en su mayoría se encontraban incluidos en el POS (paquete obligatorio de prestaciones de salud del régimen contributivo), de las cuales en gran medida eran referidas al suministro de medicamentos y a la realización de cirugías 30 .

Podemos afirmar que nuestros resultados son coincidentes con la bibliografía revisada $8,24,25,26,27,28,29,30,31$. Se observa un perfil de reclamos similar, destacándose los aumentos de cuotas, las negativas de cobertura de prestaciones y los cambios unilaterales de las condiciones del contrato. También se destaca las recurrencias por parte de las empresas de planes y seguros privados de salud al incumplimiento de la normativa existente, así como las debilidades de la regulación estatal que se expresan en el creciente proceso de judicialización, mencionado en diferentes estudios 8,24,25,26,27,29,30,31,35,36.

\section{Consideraciones finales}

A partir de lo expuesto, podemos decir que existen fuertes indicios que muestran la presencia y persistencia de problemas en la prestación de servicios de salud por parte de las empresas de medicina prepaga, que como se mostró, no es exclusivo de nuestro país.

Si bien el trabajo se centró en las empresas de medicina prepaga, para pensar la problemática abordada y las estrategias de superarla, es preciso superar las miradas fragmentadas de los sistemas de salud, así como pensar en políticas de regulación que involucren a todos los actores del sistema 8,18,23,36.

Entre las cuestiones a investigar encontramos relevante poder desarrollar estudios que releven la opinión y la experiencia de los afiliados de las empresas de medicina prepaga para conocer la calidad de las prestaciones otorgadas por las mismas. También consideramos importante la realización de estudios sobre el grado de ejercicio de los derechos frente a la vulneración de los mismos ${ }^{8}$. Tal como mencionamos al inicio del trabajo, la limitada información del sector $11,13,18,37,38$, hace que resulte fundamental contar con un sistema de información que centralice y sistematice la totalidad de las denuncias presentadas ante las diferentes agencias estatales del país, así como viabilizar el acceso público a dicha información para generar mayor transparencia. En esta línea, estudios sobre seguros privados coinciden en la importancia de contar con sistemas de información que permitan realizar un seguimiento y control adecuado, así como también para que el mismo pueda ser utilizado por los diversos gestores de salud en el proceso de toma de decisiones 8,38,39,40,41,42.

Resulta relevante advertir las dificultades que se identifican en cuanto a las capacidades de regulación en el caso de Brasil 8,23,40,42, con más de una década de vigencia de la ley que regula el sector. Bahia \& Scheffer 23 señalan entre las críticas a la agencia reguladora: su incapacidad para solucionar los problemas de los contratos antiguos, la dificultad para viabilizar el resarcimiento al Sistema Único de Salud (SUS), cuando los usuarios de planes de salud son atendidos en hospitales públicos, la no promoción de mecanismos de participación ciudadana, el mantener una política de ajuste de precios cuestionable, y por sancionar y recaudar poco a través de las multas aplicadas, entre las más destacadas 23 .

Respecto a las formas de regulación más adecuadas, en 2012, el Banco Mundial 41 publicó un documento sobre los seguros privados de salud en el que realiza una serie de recomendaciones. Entre los aspectos más relevantes se destaca el de contar con una legislación que defina con claridad las responsabilidades de la agencia de supervisión, así como la importancia de que los objetivos de la misma sean públicamente definidos para fomentar la transparencia y proveer las bases bajo las cuales el público, el gobierno, legisladores y otros actores interesados puedan evaluar su desempeño ${ }^{41}$. Se sugiere también la independencia del supervisor, y la necesidad de definir con claridad los límites otorgados a la agencia de regulación y aquellas en las que debe intervenir el poder judicial 41.

Por último, entendemos la problemática en el marco de una discusión más amplia que se inscribe en los ejes de los estudios que abordan las limitaciones observadas para la efectivización de los derechos de los ciudadanos 31,35,39 $\mathrm{y}$, en particular, aquellos que dan cuenta de las debilidades de las capacidades de las instituciones de regulación, creadas en el marco de las reformas neoliberales 5,6,7,19,43,44. En este sentido, los estudios del neoinstitucionalismo histórico también forman parte de este marco más amplio, destacando la centralidad de evaluar las políticas e instituciones, a partir del análisis de los actores vinculados al proceso de toma de decisiones, las trayectorias histórico-institucionales, 
las estrategias de los actores, como elementos claves para la comprensión de los procesos de institucionalización, cambios o mantenimiento de políticas 45 . El desafío que se nos presenta es poder crear en la sociedad, como en las diver- sas esferas del Estado, la capacidad de resistir y controlar de forma efectiva estos procesos privatizantes, defendiendo el derecho a una salud universal que se exprese y se consolide en una nueva institucionalidad.

\section{Resumo}

O artigo analisa os problemas dos usuários das empresas de planos e seguros privados de saúde na Argentina quando as mesmas não cumprem com a Lei de Defesa do Consumidor. O universo de estudo foram as reclamações dos usuários durante os anos 2000-2008 encaminhadas à Subsecretaría de Defensa del Consumidor, e as disposições e os laudos emitidos pela Subsecretaría. Um aspecto conclusivo é a recorrência do não cumprimento dos serviços do Programa Médico Obrigatório e o argumento das empresas centrado na responsabilização dos usuários. Avalia-se que a falta de um sistema de informação dificulta estudos empíricos que reflitam adequadamente o problema. Assim, com base em estudos sobre empresas de planos e seguros de saúde em outros países da América Latina, observa-se a importância de realizar estudos sobre as mesmas, sua relação com os sistemas de saúde e os processos de limitação e negação da cidadania e da crescente judicialização da saúde.

Sistemas Pré-pagos de Saúde; Legislação \& Jurisprudência; Defesa do Consumidor

\section{Colaboradores}

M. J. Luzuriaga participó de la redacción del artículo y de la revisión crítica del mismo. H. Spinelli colaboró en su estructura teórico-metodológica y revisión crítica.

\section{Agradecimientos}

A Ligia Bahia por la lectura crítica del artículo y las recomendaciones bibliográficas. 


\section{Referencias}

1. Newhouse JP. Cream skimming, asymmetric in formation and a competitive insurance market. J Health Econ 1984; 3:97-100.

2. Pauly MV. Equity and costs. Law Med Health Care 1985; 13:28-31.

3. Pauly MV, Nichols LM. 10 myths of the uninsured. AHIP Cover 2004; 45:16-20.

4. Belmartino S, Bloch C. El sector salud en Argentina: actores, conflicto de intereses y modelos organizativos, 1960-1985. Buenos Aires: Oficina Panamericana de la Salud; 1994.

5. Belmartino S. Nuevas reglas de juego para la atención médica en la Argentina ¿Quién será el árbitro? Buenos Aires: Lugar Editorial; 1999.

6. Bahia L. Padrões e mudanças das relações públicoprivado: os planos e seguros saúde no Brasil [Tesis de Doctorado]. Rio de Janeiro: Escola Nacional de Saúde Pública, Fundação Oswaldo Cruz; 1999.

7. Iriart C, Merhy E, Waitzkin H. La atención gerenciada en América Latina. Transnacionalización del sector salud en el contexto de la reforma. Cad Saúde Pública 2000; 16:95-105.

8. Scheffer M. Os planos de saúde nos tribunais: uma análise das ações judiciais movidas por clientes de planos de saúde, relacionadas à negação de coberturas assitenciais no Estado de São Paulo [Disertación de Maestría]. São Paulo: Faculdade de Medicina, Universidade de São Paulo; 2006.

9. Luzuriaga MJ. Regulación, medicina privada y ciudadanía: un estudio acercas de las denuncias presentadas ante la Subsecretaría de Defensa del Consumidor de la Nación sobre los seguros de salud de las empresas de medicina prepaga durante el periodo 2000-2008 [Disertación de Maestría]. Buenos Aires: Instituto de Salud Colectiva, Universidad Nacional de Lanús; 2012.

10. Instituto Nacional de Estadísticas y Censos. Censo Nacional de Población y Vivienda de 2010. Buenos Aires: Instituto Nacional de Estadísticas y Censos 2010.

11. Fidalgo MM. La transformación de la Seguridad Social en salud: la política de libre elección de obra social como parte de las redefiniciones del sistema de protecciones sociales. Área metropolitana de Buenos Aires (1990-2010) [Tesis de Doctorado] Buenos Aires: Facultad de Ciencias Sociales, Universidad de Buenos Aires; 2013.

12. Giovanella L, Feo O, Faria M, Tobar S. Sistemas de salud en América Latina, desafíos para la universalidad, la integralidad y la equidad. Rio de Janeiro: Instituto Sul-Americano de Governo em Saúde; 2012.

13. Repetto F, Potenza del Mazeto F. La protección social en Argentina. Santiago de Chile: División Políticas Sociales, Comisión Económica para América Latina y el Caribe; 2011. (Serie Políticas Sociales, 174).

14. Giedion U, Villar M, Ávila A. Los sistemas de salud en Latinoamérica y el papel del seguro privado. Madrid: Fundación MAPFRE; 2010.

15. Presidencia de la República Argentina. Decreto no 357, de 21 de febrero de 2002. Boletín Oficial de la República Argentina 2002; 21 feb.
16. Congreso de la República Argentina. Ley no 24.240 22 de setiembre de 1993. Instituye las normas de protección y defensa de los consumidores. Boletín Oficial de la República Argentina 1993, 15 oct.

17. Belmartino S. Una década de reforma de la atención médica en Argentina. Salud Colect 2005; 1:151-71.

18. Fidalgo M. Adiós el derecho a la salud. El desarrollo de la medicina prepaga. Buenos Aires: Espacio Editorial; 2008.

19. Iriart C. Capital financiero versus complejo médico-industrial: los desafíos de las agencias regulatorias. Ciênc Saúde Coletiva 2008; 13:1619-26.

20. Cetrángolo O, Devoto F. Organización de la salud en Argentina y equidad: una reflexión sobre las reformas de los años noventa e impacto de la crisis actual. In: Regional consultation on policy tools: equity in population health. http://www.eclac. org/publicaciones/xml/1/13041/SALUDEQYREF. pdf (accedido el 10/Jun/2013).

21. Congreso de la República Argentina. Ley no 26.682 de 4 de mayo de 2011. Instituye el marco regulatorio de medicina prepaga. Boletín Oficial de la República Argentina 2011; 17 may.

22. Frustagli SA. La protección del paciente ante las medicinas prepagas. http://revista.cideci.org/in dex.php/trabajos/article/viewFile/110/149 (accedido el 10/Jun/2013).

23. Bahia L, Scheffer M. Planos e seguros de saúde. O que todos devem saber sobre a assistência médica suplementar no Brasil. São Paulo: Editora UNESP; 2010.

24. Conte AD, Bahia L, Barroso AF. O papel da Justiça nos planos e seguros de saúde no Brasil. Cad Saúde Pública 2009; 25:279-90.

25. Machado JR. Negativas de cobertura pelas operadoras de planos de saúde: análise das denúncias de beneficiários encaminhadas à Agência $\mathrm{Na}-$ cional de Saúde Suplementar (ANS) [Disertación de Maestría]. Rio de Janeiro: Escola Nacional de Saúde Pública Sergio Arouca, Fundação Oswaldo Cruz; 2011

26. Superintendencia de Servicios de Salud de Chile. Balance de reclamos 2012. http://www.supersa lud.gob.cl/568/articles-8103_ppt_1.pdf (accedido el 05/May/2013)

27. Zamora Vergara RA. Las Isapres en Chile, su institucionalidad jurídica y el derecho a la protección de la salud: aplicación y eficacia [Monografía de Graduación]. Santiago de Chile: Facultad de Derecho, Universidad de Chile; 2012.

28. López de Maturana A, Desirée C, García Véliz RC Palacios Sovier VA. Isapres, su regulación legal y la discriminación por riesgos en la cobertura de salud [Monografía de Graduación]. Santiago de Chile: Facultad de Derecho, Universidad de Chile; 2012.

29. Yamin AE, Parra-Vera O. How do courts set health policy? The case of the Colombian Constitutional Court. PLoS Med 6(2):e1000032. 
30. Rodríguez Garavito C. La judicialización de la salud. Síntomas, diagnósticos y prescripciones. En: Bernal O, Gutiérrez C, editores. La salud en Colombia. Logros, retos y recomendaciones. Bogotá: Universidad de los Andes; 2011. p. 507-60.

31. Abramovich V, Pautassi L. El derecho a la salud en los tribunales. Algunos efectos del activismo judicial sobre el sistema de salud en Argentina. Salud Colect 2008; 4:261-82.

32. Presidencia de la República Argentina. Decreto no 1.172, del 3 de diciembre de 2003. Boletín Oficial de La República Argentina 2003; 4 dic.

33. Subsecretaría de Defensa del Consumidor de la Nación. Centro de atención telefónica gratuita. Revista Temas del Consumidor 2005; 72:64-8.

34. Corte Suprema de Justicia de la Nación. E. 34. XXXV. Recurso de hecho. Etcheverry, Roberto Eduardo c/ Omint Sociedad Anónima y Servicios. http://www.csjn.gov.ar/confal/ConsultaComple taFallos.do?method=verDocumentos\&id=499505 (accedido el 10/Jun/2013).

35. Fleury S. Judicialização pode salvar o SUS. Saúde Debate 2012; 36:159-62.

36. Sestelo J. Produção academica sobre saúde suplementar no Brasil de 2000 a 2010: revição critica e investigação sobre articulação entre o publico e o privado [Disertación de Maestría]. Salvador: Instituto de Saúde Coletiva, Universidade Federal de Bahia; 2012.

37. Belmartino S. Los servicios de atención médica. In: Torrado S, editor. El costo social del ajuste (Argentina 1976-2002): tomo II. Buenos Aires: Edhasa; 2010. p. 103-67.

38. Bahia L, Luiz RR. Informações sobre coberturas e preços de planos e seguros privados de saúde: as perspectivas da complementaridade entre fontes administrativas e de base populacional. Ciênc Saúde Coletiva 2006; 11:878-80.
39. Cunill Grau N, Fernandez MM. ¿Cómo los aspectos institucionales y organizativos inciden sobre la equidad? El caso de la política con enfoque de derechos de la salud en Chile. Revista de Ciencias Sociales 2011; 17:195-213.

40. Ocké-Reis O. O mercado de planos de saúde: o problema vira solução? Ciênc Saúde Coletiva 2008; 13:1398-400.

41. World Bank. Private voluntary health insurance: consumer protection and prudential regulation. Washington DC: World Bank; 2012.

42. Costa NR. O regime regulatorio e o mercado de planos de saúde no Brazil. Ciênc Saúde Coletiva 2008; 13:1453-63.

43. Fleury S. ¿ Universal, dual o plural? Modelos y dilemas de la atención de la salud en América Latina: Chile, Brasil y Colombia. In: Molina CG, del Arco $\mathrm{N}$, editores. Servicios de salud en América Latina y Asia. Washington DC: Banco Interamericano de Desarrollo; 2003. p. 3-37.

44. Rodríguez O. Hacia una reforma del Sistema de Seguridad Social: salud, pensiones y riesgos profesionales. Bogotá: Centro de Investigaciones para el desarrollo (CID), Facultad de Ciencias Económicas, Universidad Nacional de Colombia/Ministerio de Salud de la República de Colombia; 2002.

45. Giaimo S. Who pays for health care reform. In: Pierson P, editor. The new politics of welfare state. New York: Oxford University Press; 2001. p. 334-67.

Recibido el 24/Jul/2013

Versión final presentada el 12/Nov/2013

Aprobado el 25/Nov/2013 